\title{
Automonitorización de glucemia capilar como predictor de hemoglobina glicada
}

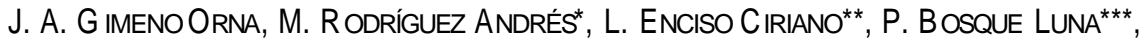 \\ B. BONED JULIANI****
}

Médico especialista en Endocrinología y Nutrición. Servicio de Endocrinología. Hospital de

Alcañiz.

*Médico Interno Residente en Medicina Familiar y Comunitaria. Centro de Salud de Alcañiz. ${ }^{* *}$ Médico especialista en Medicina Familiar y Comunitaria. ${ }^{* * \star D i p l o m a d o ~ U n i v e r s i t a r i o ~ e n ~ E n f e r-~}$

mería.

\section{RESUMEN}

Fundamento y objetivos: la automonitorización de glucemia capilar en pacientes con diabetes tipo 2 podría indicar el grado de control metabólico. Los objetivos del presente trabajo fueron evaluar en qué momento del día las glucemias capilares mostraban mejor correlación con las cifras de HbAlc y establecer puntos de corte de glucemia capilar para predecir cifras de HbAlc por encima o por debajo de 7,5\%.

Diseño: estudio descriptivo transversal.

Material y métodos: a 42 pacientes con diabetes tipo 2 se les realizaron 3 perfiles glucémicos capilares domiciliarios de 6 puntos (antes y 2 horas después de desayuno, comida y cena), el día anterior, 4 semanas antes y 8 semanas antes de la determinación de una cifra de HbAlc.

Resultados: las glucemias que mostraban mejor correlación con las cifras de HbAlc eran las de antes de desayuno $(r=0,44 ; p=0,0047)$ y las de antes de cena ( $r=0,44 ; p=0,0041)$. La curva ROC de glucemia de antes de desayuno para predecir HbAlc con un punto de corte de 7,5\% tuvo un área bajo la curva de 0,694 (IC 95\%: 0,524-0,833); el valor óptimo de glucemia elegido fue de $145 \mathrm{mg} / \mathrm{dl}$. La curva ROC de glucemia de antes de cena tuvo un área bajo la curva de 0,739 (IC 95\%: 0,574 0,866); el valor glucémico elegido fue de $141 \mathrm{mg} / \mathrm{dl}$.

Conclusiones: las glucemias que mejor correlacionan con HbAlc son las de antes de desayuno y cena. Dadas las correlaciones débiles entre HbAlc y glucemia capilar, la automonitorización debe considerarse orientativa, no sustitutiva, de la HbAlc.

Palabras clave: Automonitorización de glucemia capilar. Hemoglobina glicada.
Self monitoring of blood glucose as a predictor of glicated hemoglobin

\section{ABSTRACT}

Introduction and objectives: self monitoring of blood glucose in patients with type 2 diabetes could reflect the quality of metabolic control. The objective was to evaluate in which moments of the day capillary blood glucose showed the best correlation with glycated hemoglobin ( $\mathrm{HbAlc})$ values and to establish capillary blood glucose cut-off in order to predict HbAlc values higher and lower than $7.5 \%$.

Design: cross-over, descriptive study.

Material and methods: three capillary blood glucose profiles were practised at home to 42 patients with type 2 diabetes mellitus: one of them on the day before HbAlc determination and the other ones four and eight weeks before that HbAlc determination. Each profile included six checking points, before and two hours after breakfast, lunch and dinner.

Results: the best correlation between capillary blood glucose and HbAlc was obtained before breakfast $(r=0,44 ; p=0.0047)$ and before dinner $(r=0.44 ; p=0.0041)$. The ROC curve of blood glucose obtained before breakfast to predict HbAlc with $7.5 \%$ cut-off had an area under graph of 0.694. The optimal blood glucose value choosed was 145 $\mathrm{mg} / \mathrm{dl}$.The ROC curve of blood glucose obtained before dinner had an under graph area of 0.739; the blood glucose value choosed was $141 \mathrm{mg} / \mathrm{dl}$.

Conclusions: blood glucoses that showed the best correlation with HbAlc values were the ones before breakfast and dinner. Due to poor correlation between HbAlc and capillary blood glucoses, selfmonitoring blood glucose should be considered orientative and not sustitutive of HbAlc determination.

Key words: Self monitoring blood glucose. Glycated hemoglobin. 


\section{INTRODUCCIÓN}

La importancia del buen control metabólico de la diabetes mellitus para la prevención de las complicaciones crónicas microangiopáticas está fuera de toda duda, tanto en la diabetes tipo $1^{1}$ como en la diabetes tipo $2^{2}$.

Aunque sugerido por algunos autores ${ }^{3}$, no ha podido confirmarse la existencia de un umbral glucémico claro a partir del cual aumentase de modo marcado el riesgo de microangiopatía ${ }^{4}$.

Por ello se acepta que el control óptimo de la diabetes supondría alcanzar cifras glucémicas semejantes a las de la persona no diabética. La dificultad de alcanzar este objetivo ha hecho que el $E \mathbf{E}$ ropean Diabetes Policy Group haya recientemente acordado que un control aceptable sería alcanzar cifras de hemoglobina glicada (HbA1c) inferiores al $7,5 \%$ en los pacientes con diabetes tipo $2^{5}$.

En la práctica clínica habitual se utiliza la HbA1c como parámetro de control metabólico, la cual refleja el control de los 2-3 meses previos ${ }^{6}$. La frecuencia óptima de su determinación sería pues cada 3 meses, pero la sobrecarga asistencial ha obligado a aceptar, en pacientes con diabetes tipo 2 estable, una determinación semestral.

En la actualidad se recomienda la automonitorización de la glucemia capilar como parte fundamental del tratamiento intensivo de pacientes tipo 1 y pacientes tipo 2 insulinizados ${ }^{1}$. En pacientes tipo 2 tratados con antidiabéticos orales la automonitorización de glucemia capilar podría servir como predictora del mantenimiento o no del paciente dentro de un control metabólico aceptable.

Los objetivos del presente trabajo fueron, to mando como población diana pacientes diabéticos tipo 2 tratados con antidiabéticos orales:

-Evaluar en qué momento del día las glucemias capilares muestran mejor correlación con las cifras de $\mathrm{HbA} 1 \mathrm{c}$.

-Establecer puntos de corte de glucemia capilar para predecir cifras de $\mathrm{HbA} 1 \mathrm{c}$ por encima o por debajo de $7,5 \%$.

\section{MATERIAL Y MÉTODOS}

El estudio tuvo un diseño transversal (evaluación puntual del grado de control metabólico) con aplicación a todos los sujetos de la muestra del criterio de referencia $(\mathrm{HbA} 1 \mathrm{c})$ y del método en estudio (glucemia capilar).

Se seleccionó una muestra de pacientes con diabetes tipo 2 que cumpliesen los siguientes criterios de inclusión: edad entre 40 y 80 años, tratamiento actual con antidiabéticos orales, conocimiento previo de la técnica de automonitorización de glucemia capilar, ausencia de gestación y ausencia de otras enfermedades crónicas con afectación del estado general. Se utilizó un muestreo no probabilístico consecutivo a medida que los pacientes acudían a la consulta.

Los pacientes fueron instruidos para realizar, durante un periodo de 3 meses, una dieta estable, isocalórica en pacientes con índice de masa corporal (IMC) menor de $27,5 \mathrm{Kg} / \mathrm{m}^{2}$ e hipocalórica en pacientes con IMC superior a $27,5 \mathrm{Kg} / \mathrm{m}^{2}$. La composición de dicha dieta recomendada era 55\% hidratos de carbono, $15 \%$ proteínas y $30 \%$ grasas, de las cuales menos de la tercera parte debían ser saturadas. No se realizó modificación de la dosis de antidiabéticos orales durante el periodo de estudio.

Durante el estudio cada paciente realizó 3 perfiles glucémicos, uno de ellos el día previo a la determinación de HbA1c, otro 4 semanas antes y el tercero 8 semanas antes. Dichos perfiles constaban de 6 puntos, con glucemias antes de desayuno, comida y cena y dos horas después de desayuno, comida y cena.

La glucemia capilar se midió en domicilio mediante uno de los 5 aparatos para los que el INSALUD suministra tiras reactivas en nuestra área de salud: Glucometer Elite, Glucotouch, Glucocard Memory, Medisense precisión y Accutrend sensor. $\mathrm{La} \mathrm{HbA1c}$ se ha determinado en el analizador bioquímico Cobas mediante un inmunoanálisis turbidimétrico. El valor medio de referencia de $\mathrm{HbA} 1 \mathrm{c}$ fue del $5,1 \%$. El intervalo de referencia fue del 4,5$5,7 \%$, de acuerdo con un intervalo de confianza del $95 \%$.

Dada la escasez de datos acerca de la sensibilidad y especificidad de la glucemia capilar para predecir cifras de $\mathrm{HbA} 1 \mathrm{c}$ con la metodología aplicada en este trabajo, no se planteó la predeterminación de un tamaño muestral, por lo que puede considerarse un estudio piloto.

Se calculó la media de las tres glucemias de cada uno de los 6 puntos diarios, la media de las 9 glucemias preprandiales, la media de las 9 glucemias postprandiales y la media de las 18 glucemias de cada sujeto. Se estudió el grado de asociación line al de cada una de estas medias con la cifra de HbA1c mediante el coeficiente de correlación de Pearson, con cálculo de la recta de regresión, tomando la $\mathrm{HbA} 1 \mathrm{c}$ en cada caso como variable dependiente. Para los momentos temporales que presentaban mejor correlación con la cifra de HbA1c se calculó la curva ROC de cifras de glucemia para predecir una HbA1c superior o inferior al 7,5\%.

Se utilizó programa informático MedCalc para Windows.

\section{RESULTADOS}

Se seleccionaron 50 pacientes que cumplían los criterios de inclusión, todos los cuales aceptaron 
participar. Se excluyeron 8 de ellos por no aportar el número planteado de determinaciones de glucemia capilar. Así, la muestra final fue constituida por 42 pacientes, de los cuales 20 eran varones y 22 mujeres. La edad media era de 64,5 $\pm 7,5$ años y el tiempo de evolución medio conocido de la enfermedad de $9,2 \pm 5,8$ años. El IMC medio era de $29 \pm 5,8 \mathrm{Kg} / \mathrm{m}^{2}$. De los pacientes había un $88 \%$ en tratamiento con sulfonilureas, un $78,6 \%$ en tratamiento con metformina y un $21,5 \%$ en tratamiento con acarbosa.

La cifra media de HbA1c era 7,4 $40,89 \%$. Las cifras medias de glucemia capilar en cada momento temporal, su coeficiente de correlación con HbA1c y su recta de regresión se reflejan en la tabla I. Las glucemias que mostraban mejor correlación con las cifras de HbA1c eran las de antes de desayuno ( $r=0,44$ (IC 95\%: 0, 16-0,65); $\mathrm{p}=0,0047$ (Fig. 1) y las de antes de cena ( $\mathrm{r}=0,44$ (IC 95\%: 0,16-0,65); $\mathrm{p}=0,0041$ (Fig. 2).

Tabla I

VALORES MEDIOS Y DESVIACIÓN ESTÁNDAR (DE)
DE GLUCEMIA CAPILAR EN LOS DIFERENTES
MOMENTOS DEL DÍA, CORRELACIÓN CON H bA1C

\begin{tabular}{lllll} 
MOMENTO & MEDIA DE & $R$ & RECTA \\
\hline Predesayuno & 136 & 30,1 & $0,44^{*}$ & $y=5,6+0,013 x$
\end{tabular}

$\begin{array}{lllll}\text { Posdesayuno } & 171 & 35,6 & 0,31 & y=6,1+0,008 x\end{array}$

$\begin{array}{lllll}\text { Precomida } & 136 & 27,6 & 0,11 & y=7+0,0034 x\end{array}$

$\begin{array}{lllll}\text { Poscomida } & 166 & 42,3 & 0,21 & y=6,7+0,0046 x\end{array}$

$\begin{array}{lllll}\text { Precena } & 133 & 35,2 & 0,44 * & y=5,9+0,011 x\end{array}$

$\begin{array}{lllll}\text { Poscena } & 165 & 36,3 & 0,26 & y=6,4+0,0062 x\end{array}$

Preprandiales $\quad \begin{array}{llll}136 & 24,9 & 0,42 * & y=5,4+0,015 x\end{array}$

$\begin{array}{lllll}\text { Postprandiales } \quad 170 & 30,4 & 0,28 & y=6+0,0085 x\end{array}$

\begin{tabular}{lllll}
\hline Total & 154 & 25,4 & $0,34 * *$ & $y=5,7+0,012 x$ \\
\hline
\end{tabular}

${ }^{*} p<0,01 ;{ }^{* *} p<0,05$

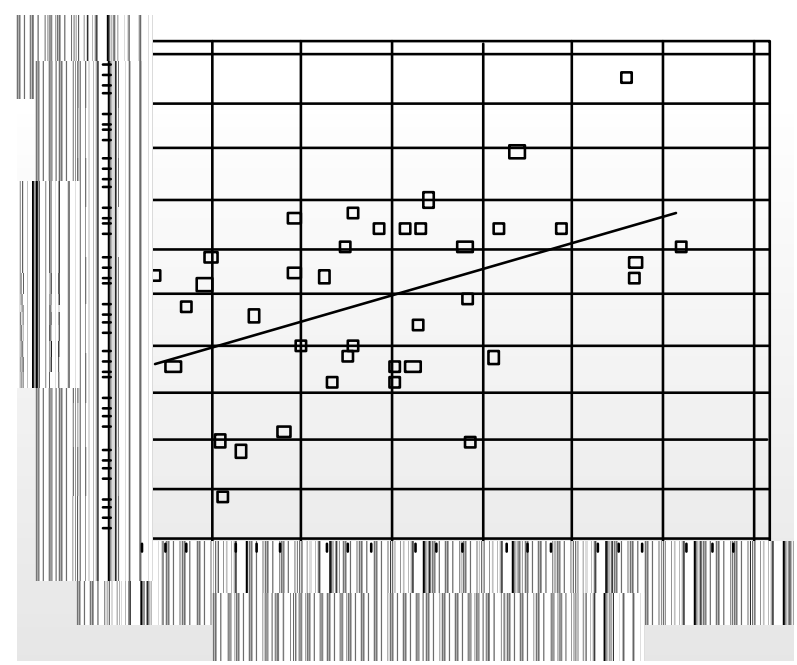

Figura 1

Correlación entre glucemia predesayuno y $\mathrm{HbA} 1 \mathrm{c}$

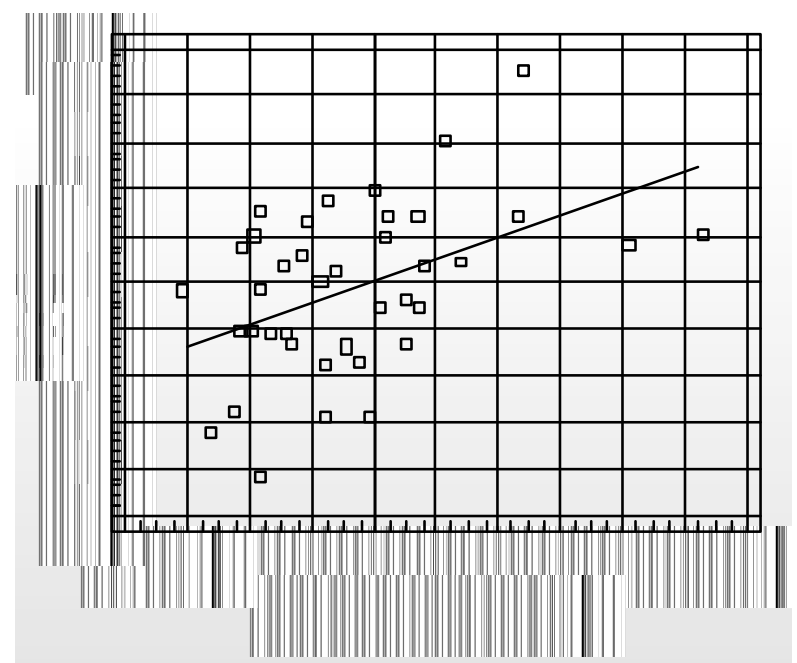

Figura 2

Correlación entre glucemia precena y $\mathrm{HbA} 1 \mathrm{c}$

La curva ROC de glucemia de antes de desayuno para predecir $\mathrm{HbA} 1 \mathrm{c}$ con un punto de corte de 7,5\% tuvo un área bajo la curva de 0,694 (IC 95\%: 0,524-0,833). El valor óptimo de glucemia elegido por el programa informático fue de $145 \mathrm{mg} / \mathrm{dl}$, con sensibilidad (S) del 50\% (IC 95\%: 35-65\%), especificidad (E) del 89\% (IC 95\%: 80-98\%), valor predictivo positivo (VPP) del $83 \%$ (IC 95\%: 72$94 \%$ ) y valor predictivo negativo (VPN) del $61,5 \%$ (IC 95\%: 47-76\%) (Fig. 3). Descender el valor de glucemia a $120 \mathrm{mg} / \mathrm{dl}$ supuso un aumento de la $\mathrm{S}$ hasta un $75 \%$ (IC 95\%: 62-88\%) y una pérdida de E hasta un 50\% (IC 95\%: 35-65\%), con VPP $62,5 \%$ (IC 95\%:48,5-76,5\%) y VPN 64\% (IC 95\%: $49,5-78,5 \%)$.

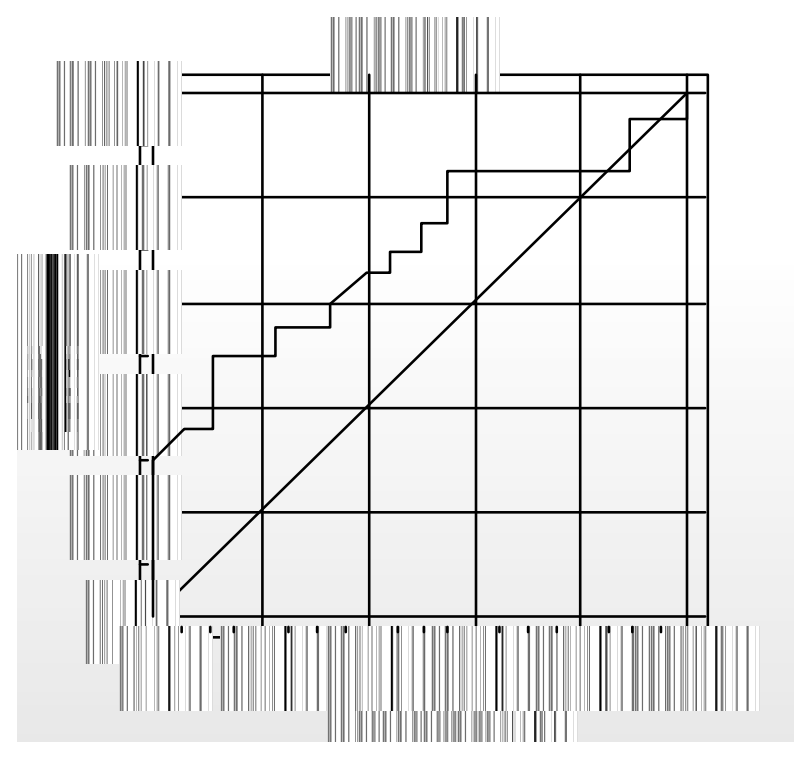

Figura 3

Curva ROC de glucemia predesayuno (glprde) para predecir $\mathrm{HbA} 1 \mathrm{c}$ con punto de corte de 7,5\% 
La curva ROC de glucemia de antes de cena tuvo un área bajo la curva de 0,739 (IC 95\%: 0,574$0,866)$. El valor glucémico elegido fue de 141 $\mathrm{mg} / \mathrm{dl}$, con S del 55\% (IC 95\%: 40-70\%), E del 84,2\% (IC 95\%: 73-95\%), VPP del 78,6\% (IC 95\%: $66-91 \%$ ) y VPN del 64\% (IC 95\%: 49,5-78,5\%) (Fig. 4). El punto de corte de $120 \mathrm{mg} / \mathrm{dl}$ tuvo una S de un $70 \%$ (IC 95\%: 56-84\%) y E del 53\% (IC 95\%: 38-68\%), con VPP de 61\% (IC 95\%: 46,5$75,5 \%$ ) y VPN de $62,5 \%$ (IC 95\%: 48-77\%).

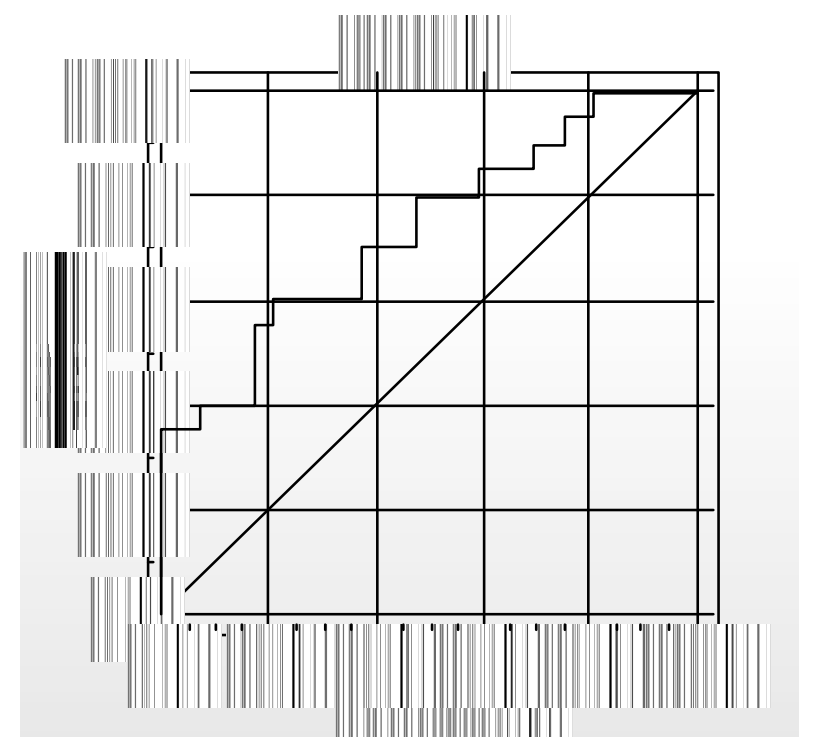

Figura 4

Curva ROC de glucemia precena (glprce) para predecir $\mathrm{HbA} 1 \mathrm{c}$ con punto de corte de $7,5 \%$

\section{DISCUSIÓN}

En el presente trabajo hemos obtenido correlaciones débiles, inferiores a 0,5 , entre las cifras de glucemia obtenidas por automonitorización de glucemia capilar y la cifra de HbA1c determinada en el laboratorio.

En el estudio NHANES III ${ }^{7}$, representativo de la población general americana, pudo comprobarse que la prevalencia de retinopatía aumentaba brus camente en el último decil de la distribución de glucemia basal venosa y HbA1c de la población. Los sujetos de este decil tenían una glucemia basal venosa media de $165 \mathrm{mg} / \mathrm{dl}$ (rango de 120-446) y una $\mathrm{HbA} 1 \mathrm{c}$ media de 7,4\% (rango de 6,2 a 15,7\%). Por lo tanto parece razonable establecer un objetivo de control glucémico óptimo centrado en una HbA1c menor a 6,2\% y un control aceptable en una $\mathrm{HbA} 1 \mathrm{c}$ inferior a 7,5\%.

La glucemia plasmática basal en sujetos tratados con antidiabéticos orales se ha revelado un indicador imperfecto de control glucémico en diferentes estudios. Así El Kebbi et $\mathrm{al}^{8}$ comunicaron que una glucemia basal venosa de $165 \mathrm{mg} / \mathrm{dl}$ predecía una $\mathrm{HbA} 1 \mathrm{c}>8 \%$ con $\mathrm{S}$ del $79 \%$, E del $78 \%$ y VPP del $78 \%$. En el estudio de Bouma et al $^{9}$ la glucemia venosa basal y la HbA1c alcanzaron una correlación de 0,77 , con una cifra de $140 \mathrm{mg} / \mathrm{dl}$ aportando una $\mathrm{S}$ del $90 \%$ y una $\mathrm{E}$ del $66 \%$ para predecir una $\mathrm{HbA} 1 \mathrm{c}>7 \%$. En un estudio francés ${ }^{10}$ la glucemia venosa de antes de la cena y la de después de la comida fueron mejores predictores de control glucémico que la glucemia basal, con coeficientes de correlación respectivos de 0,73, 0,84 y 0,6.

Ha habido pocos estudios correlacionando perfiles glucémicos capilares con HbA1c. En el DCCT ${ }^{1}$ el grupo de tratamiento intensivo tuvo una glucemia capilar media total de $155 \mathrm{mg} / \mathrm{dl}$ y una HbA1c de $7,1 \%$; en nuestro trabajo la glucemia media total fue la misma, pero la HbA1c fue ligeramente superior, diferencias posiblemente atribuibles a que en el DCCT los pacientes eran tipo 1 y a la diferente metodología de determinación de HbA1c. En un estudio catalán de Atención Primaria recientemente publicado ${ }^{11}$ se describió la existencia de una baja concordancia entre las cifras de HbA1c y los perfiles de glucemia capilar; en nuestros pacientes la correlación entre ambas también fue débil.

Hay que tener en cuenta factores que hayan podido influir en las bajas correlaciones obtenidas. En primer lugar se utilizaron diferentes autoanalizadores en los pacientes. Se ha descrito ${ }^{12}$ que las cifras de glucemia capilar son hasta un $14 \%$ inferiores a las venosas, aunque determinados autoanalizadores como Glucometer Elite y Medisense podrían dar valores más próximos a los de glucemia plasmática. Al menos cada paciente utilizó siempre el mismo aparato. En segundo lugar no puede descartarse una diferente adherencia a la dieta los días de realización del perfil glucémico. Este sesgo vendría motivado por el deseo del paciente de mostrar a su médico buenos perfiles glucémicos. Debemos destacar que, aunque los problemas anteriores hayan podido disminuir la validez interna del trabajo, la realización del mismo en condiciones semejantes a las de la práctica clínica habitual y en una muestra que creemos puede considerarse representativa de pacientes con diabetes tipo 2 en tratamiento con antidiabéticos orales, aumenta su validez externa (generalizabilidad).

Otro punto de controversia es si es más útil la información pre o la postprandial. Se han observado mejores correlaciones de la $\mathrm{HbA} 1 \mathrm{c}$ con las glucemias postprandiales ${ }^{12}$, especialmente en sujetos con cifras de HbA1c cerca del rango normal. En nuestro estudio y en ${ }^{11}$ la correlación fue mejor con las cifras preprandiales; esto podría justificarse por la mayor variabilidad de la cifras postprandiales, como reflejado por desviaciones estándar más amplias. Otros factores que pueden hacer variar la im- 
portancia relativa de las cifras pre o postprandiales serían el tiempo de evolución de la enfermedad y el tipo de antidiabético oral. Nuestros pacientes tenían un tiempo de evolución prolongado y la mayoría llevaba tratamiento simultáneo con sulfonilureas y metformina.

De cualquier modo es importante obtener información de las cifras de glucemia postprandial, pues se ha descrito que los picos de hiperglucemia pueden aumentar el estrés oxidativo y activar la proteína kinasa $\mathrm{C}$, favoreciendo la aparición de complicaciones crónicas ${ }^{13}$.

En el presente trabajo las glucemias de antes de desayuno y cena que mejor predecían un control aceptable o deficiente eran en torno a $140 \mathrm{mg} / \mathrm{dl}$. A partir de las rectas de regresión podía calcularse que una $\mathrm{HbA} 1 \mathrm{c}$ de 7,5\% correspondía a una glucemia predesayuno de $146 \mathrm{mg} / \mathrm{dl}$ y a una glucemia precena de $145 \mathrm{mg} / \mathrm{dl}$. El área bajo la curva ROC fue pequeña, aunque estadísticamente significativa, indicando que la glucemia capilar predice de modo imperfecto el grado de control metabólico. En el análisis de la curva ROC hubo, para los valores glucémicos elegidos como óptimos por el programa informático, una baja sensibilidad y una elevada especificidad. Dado que en este caso interesa más obtener una buena sensibilidad, detectando mediante la monitorización de glucemia capilar al mayor número posible de sujetos con un control metabólico deficiente, podría ser más útil descender el umbral glucémico hasta aproximadamente $120 \mathrm{mg} / \mathrm{dl}$. Los valores predictivos se mantuvieron en ambos casos dentro de valores aceptables.

La principal limitación del estudio fue el escaso tamaño de la muestra, lo que se tradujo en intervalos de confianza amplios. No obstante, creemos que resulta útil, dada la representatividad de la muestra.

Podemos concluir de todo lo expuesto lo siguiente:

-Las glucemias que mejor correlacionan con HbAlc son las de antes de desayuno y cena.

- Cifras de glucemia predesayuno y cena superiores a $120 \mathrm{mg} / \mathrm{dl}$ tienen una sensibilidad de un 75 y un $70 \%$ respectivamente para detectar una HbA1c mayor de 7,5\%.

-Dadas las correlaciones débiles entre HbA1c y glucemia capilar, la automonitorización debe considerarse orientativa, no sustitutiva, de la HbA1c.

\section{CORRESPONDENCIA:}

J. A. Gimeno Orna

C/ Balbino Orensanz, 19-2 ${ }^{\circ}-\mathrm{B}$

50014 Zaragoza

Telf. 97.6395629

\section{Bibliografía}

1. The DCCT Research Group. The effect of intensive treatment of diabetes on the development and progression of long term complications in insulin dependent diabetes mellitus. N Engl J Med 1993; 329: 97786.

2. UKPDS Group. Intensive blood glucose control with sulphonylureas or insulin compared with conventional treatment and risk of complications in patients with type 2 diabetes (UKPDS 33). Lancet 1998; 352: 837-53.

3. Krolewski AS, Laffel LMB, Krolewski M, Quinn M, Warram JH. Glycosylated hemoglobin and the risk of microalbuminuria in patients with insulin dependent diabetes mellitus. N Engl J Med 1995; 332: 1251-5.

4. The DCCT Research Group. The relationship of glycemic exposure $(\mathrm{HbA1c})$ to the risk of development and progression of retinopathy in the DCCT. Diabetes 1995; 44: 968-83.

5. European Diabetes Policy Group. A desktop guide to type 2 Diabetes Mellitus. Diabetic Medicine 1999; 16.

6. Equipo de trabajo del Programa del Adulto. Diabetes Mellitus. Programas Básicos de Salud. semFYC: Doyma, 1998.
7. Davidson MB, Schriger DL, Peters AL, Lorber B. Relationship between fasting plasma glucose and glycosylated hemoglobin. JAMA 1999; 281:1203-10.

8. El-Kebbi IM, Ziemer DC, Gallina DL, Phillips LS. Diabetes in urban african-americans VI. Utility of fasting or random glucose in identifying poor glycemic control. Diabetes Care 1998; 21: 501-5.

9. Bouma M, Dekker JH, De Sonnaville JJ, Van der Does FE, De Vries H, Kriegsman DM, et al. How valid is fasting plasma glucose as a parameter of glycemic control in non-insulin-using patients with type 2 diabetes? Diabetes Care 1999; 22: 4-907.

10. Avignon A, Radauceanu A, Monnier L. Nonfasting plasma glucose is a better marker of diabetic control than fasting plasma glucose in type 2 diabetes. Diabetes Care 1997; 20: 1822-6.

11. Carrión E, Flores M, Muñoz MA. Analisis de la concordancia entre la hemoglobina glucosilada y la automonitorización de la glucemia capilar: ¿puede una sustituir a la otra? Endocrinología y Nutrición 2000; 47: 133-5.

12. Buse JB, Hroscikoski M. The case for a role for postpandrial glucose monitoring in diabetes management. 